\title{
XXème CONGRES DE LA SALF
}

\section{Les 11, 12 et 13 DECEMBRE 2003 ; Centre de Conférences - ORLEANS}

\section{PRE-PROGRAMME}

\section{Mercredi 10 décembre 2003}

$14 \mathrm{~h} 30-17 \mathrm{~h} 30 \quad$ Initiation à l'Andrologie Session parrainée par Astra-Zeneca

Modérateurs : Jean-Louis Pariente (Bordeaux) / Pierre Bondil (Chambéry)

14h30 Pratique de l'androgénothérapie :

15h10 Les différentes techniques d'Assistance Médicale à la Procréation :

15h50 Pause

16h10 Prise en charge d'une azoospermie :

16h50 Bilan d'une dysfonction érectile :
Hervé Lejeune (Lyon)

Paul Barrière (Nantes)

Jean-Louis Pariente

(Bordeaux)

Pierre Bondil (Chambéry)

\section{Jeudi 11 décembre 2003}

9h - $12 \mathrm{~h} 30$ Troubles de l'éjaculation

Modérateurs : Jacques Buvat (Lille) / Jean Hermabessière (Clermont-Ferrand)

9h Introduction et Epidémiologie :

Jacques Buvat (Lille)

9h15 Physiologie de l'éjaculation :

Jean Hermabessière

(Clermont-Ferrand)

9h35 Contrôle neurologique :

Olivier Rampin

(Jouy en Josas)

9h55 Physiopathologie et traitement pharmacologique de l'éjaculation prématurée (anglais) :

Marcel Waldinger

(La Haye, Pays-Bas)

10h25 Pause, visite de l'exposition et des posters

10h55 Diagnostic d'une anéjaculation :

Antoine Lemaire (Lille)

$11 \mathrm{~h} 15$ Prise en charge des troubles de l'éjaculation des neurologiques :

Guy Egon (Le Mans)

11 h40 Ejaculation rétrograde, nombre et qualité des spermatozoïdes récupérés, utilisation en AMP :

Jacques Auger (Paris)

$12 \mathrm{~h} 05$ Sexothérapies :

Marie Chevret-Méasson (Lyon)

12h35 - 13h50 Symposium-déjeuner Pfizer :«Diabète, dysfonction endothéliale et troubles de l'érection» Président : Jacques Buvat (Lille) 
Modérateurs : Roger Mieusset (Toulouse) / Clément Jimenez (Dijon)

14h Vasectomie :

14h25 Contraceptions hormonales : aujourd'hui et demain (anglais):

14h55 Contraceptions non hormonales : aujourd'hui et demain :

$15 \mathrm{~h} 20$ Contraception épididymaire : état des recherches et perspectives :
Yves Lanson (Tours) Frederic Wu (Manchester) Louis Bujan (Toulouse) Joël Drevet (Clermont-Fd)

$15 \mathrm{~h} 45$ Pause, visite de l'exposition et des posters

16h15 - 17h15 Présentation des Bourses SALF 2002

Modérateurs : Jacqueline Lornage (Lyon) / Olivier Rampin (Jouy en Josas)

$16 \mathrm{~h} 15$ Aptitude des cellules germinales males immatures ou différenciées in vitro à promouvoir un développement embryonnaire normal :

Nada Borghol (Lyon)

$16 \mathrm{~h} 35$ Etude du contrôle de la stéroïdogenèse testiculaire humaine par les endozépines

Céline Duparc (Rouen)

$16 \mathrm{~h} 55$ Exposition combinée à la vinclozoline et à la génistéine à faibles doses de la conception à l'âge adulte : effets sur la fonction de reproduction chez le rat male de 1ère et de 2ème génération et possibles mécanismes d'action : Florence Eustache (Paris)

17h15 - 18h30 Symposium Lilly :»Dysfonction érectile : optimisation des pratiques et des traitements» Président : Albert Leriche (Lyon)

18h30 Assemblée générale de la SALF

20h30 Dîner parrainé par Lilly

\section{Vendredi 12 décembre 2003}

8h30 - 9h45 Pathologies du veru montanum et du carrefour vésiculo-déférentiel

Modérateurs : Michel Peneau (Orléans) / Vincent Izard (Paris)

$8 \mathrm{~h} 30$ Anatomie :

Vincent Delmas (Paris)

8 h55 Obstructions des canaux éjaculateurs. Diagnostic et traitements :

Laurent Wagner (Nîmes)

9h20 Pathologies du veru montanum et du carrefour vésiculo-déférentiel en dehors des obstructions :

Alain Jardin (Kremlin-Bicêtre)

9h45 Pause, visite de l'exposition et des posters

10h15 - 12h Prise en charge des cryptozoospermies et des oligospermies extrêmes

Modérateurs : Geneviève Grizard (Clermont-Ferrand) / Medhi Benchaid (Lyon)

10h15 Définition et présentation d'un travail collaboratif :

Ethel Szerman (Caen)

10h30 Bilans spécifiques et facteurs prédictifs :

Paul Barrière (Nantes)

10h50 Modalités techniques de récupération des spermatozoïdes et congélation:

Martine Albert (Poissy)

11 h10 Résultats en ICSI et issues de grossesses :

Marc Bailly (Poissy)

11h30 Table ronde «Amélioration de la prise en charge des patients présentant une cryptozoospermie» animée par

Vincent Izard (Paris) et Roger Mieusset (Toulouse)

12h00 - 12h30 Présentation des lauréats des Bourses SALF 2003 
12h35 - 13h50 Conférence-déjeuner «La sexualité en est-elle à l'âge de pierre ?» Michel Peneau (Orléans)

14h - 14h 30 Présentation des Mémoires (DEA, DESS) primés par la SALF

$14 \mathrm{~h} 30$ - $15 \mathrm{~h} 30$ Présentation orale des 6 posters sélectionnés et primés

15h30 Pause, visite de l'exposition et des posters

16h - 18h15 Prostatites chroniques et syndrome douloureux pelvien chronique de l'homme

Modérateurs : Jean-Pierre Mignard (Saint Brieuc) / Philippe Mangin (Nancy)

$16 \mathrm{~h}$ Introduction, nouvelle classification et épidémiologie :

Jean-Pierre Mignard (Saint-Brieuc)

16h15 Distinction prostatites chroniques/prostatites aiguës récidivantes :

Jean Michel Benoît (Toulouse)

16h35 Symptomatologie, retentissement sexuel et qualité de vie :

Béatrice Cuzin (Lyon)

17h Microbiologie et antibiothérapie des prostatites chroniques bactériennes : Adel Ben Ali (Paris)

17h20 Conséquences des prostatites chroniques sur le sperme et la fertilité :

Wolfang Weidner

(Giessen, Allemagne)

sous réserve

17h50 Physiopathologie et traitement du syndrome douloureux pelvien chronique de l'homme :

Christian Baude (Lyon)

18h15 - 19 h30 Symposium Bayer-GSK : «Dysfonction érectile : retrouver la puissance !» Président : Pierre Costa (Nîmes)

20h30 Dîner parrainé par Bayer-GSK

Samedi 13 décembre 2003

9h00 - 12h45 Andrologie de l'homme vieillissant

Modérateurs : Hervé Lejeune (Lyon) / Laurent Wagner (Nîmes)

1ère partie : Déficit androgènique lié à l'âge (DALA)

9h00 Introduction :

Hervé Lejeune (Lyon)

9h05 Diagnostic du DALA :

Jean-Jacques Legros

(Liège, Belgique)

9h30 L'atrophie sénile de la verge : un signe clinique de DALA ? :

Pierre Bondil (Chambéry)

9h45 Que faut-il attendre d'une androgénothérapie ?:

Jean Marc Kuhn (Rouen)

$10 \mathrm{~h} 05$ Androgènes et cancer de la prostate :

Pär Stattin (Lyon / Umea, Suède)

10h30 Surveillance prostatique d'une androgénothérapie :

Jean-Louis Pariente (Bordeaux)

10h45 Pause, visite de l'exposition et des posters

\section{$2^{\text {ème }}$ partie : Fertilité de l'homme vieillissant}

11 1515 Epidémiologie : données actuelles :

$11 \mathrm{~h} 35$ Evolution des caractères spermatiques avec l'âge :

11 h55 Age paternel et AMP :

$12 \mathrm{~h} 15$ Age paternel et risque génétique :

$12 \mathrm{~h} 35$ Conclusion et synthèse :

$12 \mathrm{~h} 45$ Clôture du Congrès - Présentation du Congrès 2004 (Clermont-Ferrand)
Patrick Thonneau (Toulouse)

Louis Sibert (Rouen)

Elise De La Rochebrochard

(Kremlin-Bicêtre)

Maurice Auroux (Paris)

Laurent Wagner (Nîmes) 


\section{INFORMATIONS GENERALES}

- Comité d'organisation :Dominique Delavierre, Laurent Corcia, Hussein Ibrahim, bdelhak Medallel, Michel Peneau

- Lieu du Congrès Centre de Conférences d'Orléans 9 Place du 6 juin 194445000 Orléans www.orléans-gestion.fr/conferences

- Certification Formation médicale continue (FMC)

Le Congrès de la SALF est certifié FMC par l'Association Française d'Urologie (AFU) (coefficient B22).

- Publication des communications

Chaque communication fera l'objet d'une publication dans la revue ANDROLOGIE

- Parrainage de la SALF

La SALF attribue des bourses, des prix DEA, DESS et Posters grâce au soutien des laboratoires Besins (partenaire depuis 2003), Lilly (partenaire depuis 2003) et Pfizer (partenaire depuis 1999).

\section{APPEL A PROPOSITION DE POSTERS}

- Les propositions de posters doivent être envoyées sous forme de résumé au secrétariat scientifique :

Dr Dominique Delavierre - Service Urologie-Andrologie - CHR La Source - BP 6709 - 45067 Orléans cedex 2 - Mail delav.uro@wanadoo.fr

- Date limite : 31 Octobre 2003

- Consignes de rédaction des résumés : format papier ou numérique PC, 1 page maximum, A4, Word, Police Times New Roman, Taille 12, Marges 2,5 cm

- Sur le résumé l'auteur qui présentera le poster doit être souligné.

- Les posters seront affichés pendant toute la durée du Congrès.

- Au cours du Congrès un jury sélectionnera les 6 meilleurs posters pour une présentation orale de 7 minutes le vendredi 12 décembre 2003 de $14 \mathrm{~h} 30$ à $15 \mathrm{~h} 30$ suivie d'une remise de prix pour chaque poster sélectionné. Dans l'hypothèse d'une sélection il sera nécessaire de prévoir une présentation de 5 diapositives maximum, numérique, formatée pour PC, enregistrée sous Power point sur support disquette ou CD-rom.

- Un poster ne pourra être affiché et participer à la sélection que si son présentateur est inscrit et présent au Congrès.

\section{RENSEIGNEMENTS}

SECRETARIAT SCIENTIFIQUE

Dr Dominique DELAVIERRE Service d'UROLOGIE-ANDROLOGIE

CHR La Source BP 670945067 ORLEANS Cedex 2 (France)

Téléphone $33(0) 238514626$ Télécopie $33(0) 238514159$ Mail delav.uro@ wanadoo.fr

\section{SECRETARIAT ADMINISTRATIF}

ORLEANS CONGRES / SALF 2003

1 rue du Président R. SCHUMAN 45074 ORLEANS Cedex (France)

Téléphone 33 (0)2 $38569730 \quad$ Télécopie 33 (0)2 38569735

Mail OrleansCongres@ orleansgestion.fr

SITE de la SALF

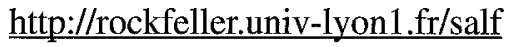

